\title{
ICTIOFAUNA DE LA LOCALIDAD DE SAN GERARDO DE LIMONCITO, FORMACIÓN CURRÉ, MIOCENO SUPERIOR, CANTÓN DE COTO BRUS, PROVINCIA DE PUNTARENAS, COSTA RICA
}

\author{
César A. Laurito ${ }^{1 *} \&$ Ana L. Valerio ${ }^{2}$ \\ ${ }^{1}$ CENETUR, Instituto Nacional de Aprendizaje. Apdo 203-2200, Coronado; \\ San José, Costa Rica \\ ${ }^{2}$ Departamento de Historia Natural, Museo Nacional de Costa Rica. Apdo \\ 749-1000, San José, Costa Rica \\ *Autor para contacto: cesarlaurito@ice.co.cr
}

(Recibido: 5/05/2008; aceptado: 12/12/2008)

\begin{abstract}
Remains of selachians (sharks and rays) and other icthiolites have been collected from the Upper Miocene locality of San Gerardo of Limoncito. Three new records of fossil species of sharks are described for Central America and the Caribbean Region: Carcharhinus longimanus (Poey, 1861), Isogomphodon caneullensis (Cappetta, 1970) and Sphyrna arambourgi (Cappetta, 1970). The fauna of the Curré Formation is notable for the strong Tethisian affinity with the paleomediterranean faunas.

Keywords: Chondrichthyes, Osteichthyes, stratigraphy, sedimentology, Curré Formation, Upper Miocene, Costa Rica.

RESUMEN: Restos de seláceos (tiburones y rayas) y otros ictiolitos fueron recolectados de la localidad de San Gerardo de Limoncito de edad Mioceno Superior. Se describen tres nuevos registros de especies fósiles de tiburones para América Central y la Región Caribe: Carcharhinus longimanus (Poey, 1861), Isogomphodon caneullensis (Cappetta, 1970) y Sphyrna arambourgi (Cappetta, 1970). La fauna de la formación Curré se caracteriza por su fuerte afinidad Testisiana con las faunas paleomediterráneas.

Palabaras clave: Chondrichthyes, Osteichthyes, estratigrafía, sedimentología, Formación Curré, Mioceno Superior, Costa Rica
\end{abstract}




\section{INTRODUCCIÓN}

La localidad fosilífera San Gerardo de Limoncito, constituye el yacimiento de vertebrados terrestres más importante del país. Entre los grupos de vertebrados superiores recuperados, destacan los reptiles representados por cocodrilos, falsos gaviales y tortugas, y los mamíferos representados por los xenarthras, camélidos, équidos, tayasuidos, mastodontes y en mucho menor grado los cetáceos.

A lo largo de cuatro años de campañas paleontológicas y producto del detallado tamizado de los sedimentos se logró recuperar algunos vertebrados inferiores, destacándose una colección de 89 dientes orales y dérmicos de peces cartilaginosos y dos dientes de peces óseos, objeto del presente estudio. También se recuperó una importante colección de restos de bagres que serán oportunamente descritos por aparte.

La localidad de San Gerardo de Limoncito, se ubica 14 kilómetros al oeste de la ciudad de San Vito (851'19.6" N y 8304'51.9”'W), cantón de Coto Brus en la Provincia de Puntarenas, Costa Rica (Fig. 1).

\section{GEOLOGÍA Y SEDIMENTOLOGÍA}

Los sedimentos de la Formación Curré, fueron inicialmente descritos como una unidad de la Formación Térraba y Dengo (1962, p. 43) fue quién de manera preliminar usó el nombre Curré "para distinguir un conjunto de rocas sedimentarias, principalmente areniscas, que afloran en el Río Térraba bajo la Formación Paso Real entre el caserío Curré y la Escuadra". Aunque Dengo (1962), no fue muy claro al momento de definir el rango estratigráfico de esta secuencia sedimentaria, si utiliza la palabra "formación" para referirse a ella; sin embargo, existe cierta ambigüedad en cuanto al uso del término "formación" por parte de este autor, pues en ningún momento explicitó que estaba definiendo a la Formación Curré, aunque si da una descripción y menciona un espesor de 830 metros para los sedimentos.
Henningsen (1965, p. 22), fue el primero en hacer referencia a la Formación Curré (sensu lato) y señala a Dengo (1962) como responsable de elevar a rango de formación la secuencia sedimentaria que él consideraba, siguiendo la interpretación de Lohmann (1934) como propios de la Formación Gatún, a pesar que el mismo Henningsen (1965) no pudo localizar el afloramiento del Río Concepción, abundante en moluscos y ubicado en "la Carretera Interamericana al oriente del Palmar en el puente sobre el río Curré", sobre el cual Lohmann (1934) fundamentó su interpretación para asignar dichos sedimentos como pertenecientes a la Fm. Gatún de edad Mioceno Medio.

Posteriormente, Mora (1979) describió dos unidades:

A) La Subunidad de conglomerados de origen volcaniclásticos de color verde grisáceo, que es sobreyacida por las lutitas.

B) La subunidad de lutitas de color negro, intercaladas con estratos de areniscas y conglomerados finos, cloritizadas, que Mora (1979) considera un producto subvolcánico. Su espesor varía entre los 150 y 300 metros.

En realidad el color verdoso de las lutitas, según lo observado por nosotros en el campo, se debe a la abundancia de glauconita que se encuentra en las arcillas y el negro se debe al detritus carbonoso que lamina estas arcillas azules.

En el caso del material paleontológico recuperado, proviene de un nivel de conglomerado fino que se ubica hacia el tope de la secuencia de las arcillas azules, que a su vez es sobreyacido por una secuencia de areniscas medias a finas. Lo que confirma su asociación con la subunidad superior o subunidad de lutitas (sensu Mora, 1979).

A continuación se realiza la descripción de las litofacies observadas y se interpreta su sedimentología.

La sedimentología de la localidad de Limoncito, se interpreta a partir de las litologías dominantes dentro de los diferentes bancos sedimentarios, de las estructuras sedimentarias y de las ichnofacies asociadas a dichas litofacies, así como de la fauna encontrada. Ello permitió establecer las siguientes facies sedimentarias (Fig. 2). 

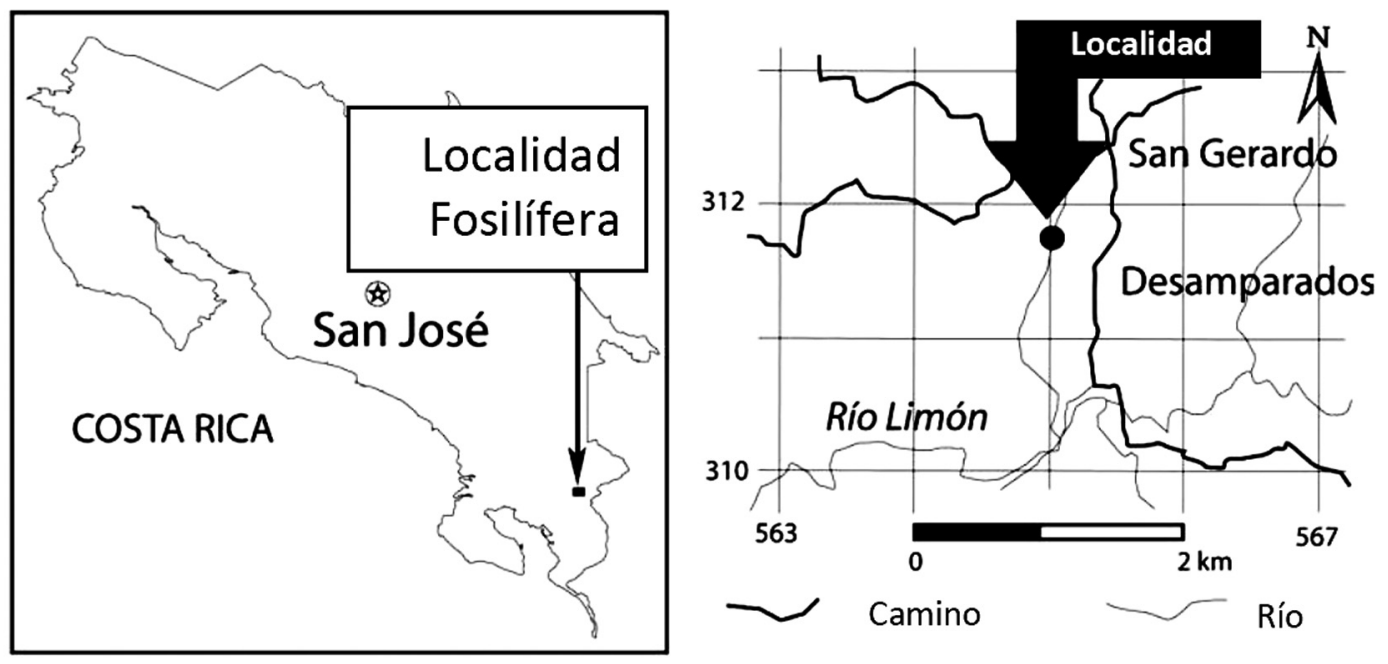

Fig. 1: Mapa de ubicación de la Localidad de San Gerardo de Limoncito, Cantón de Coto Brus.

\section{A) Plataforma Distal (Offshore)}

La litofacies de arcilla azul glauconítica es la que caracteriza este ambiente y sugiere una lenta tasa de sedimentación. Esta litofacies está constituida por un banco de 2 metros de espesor visible, de estratos decimétricos de arcillas y limos, finamente laminados con abundante detritus vegetal y madera retrabajada por el oleaje y perforada por Teredo $s p$.

No se observan trazas fósiles, lo que aunado a la presencia abundante de glauconita y material carbonoso se interpreta como un ambiente predominantemente reductor (Chafetz \& Reid, 2000).

\section{B) Abanico deltaico submareal (Fan Delta)}

La litofacies de conglomerados es la que caracteriza este ambiente y está compuesta por intraclastos de arcilla azul, procedentes del nivel infrayacente al que sobreyace mediante una discordancia erosiva. Los clastos tienen diámetros que varían entre los 5 y $13 \mathrm{~cm}$; estos son bien redondeados, poco o nada esféricos, algunos presentan contacto puntual, pero el $90 \%$ de ellos flotan en una matriz de arcilla glauconítica. Los clastos se disponen con su eje largo paralelo al plano de estratificación y es posible observar algún grado de imbricación.
En esta secuencia son frecuentes los restos óseos y dentales de diversos vertebrados tanto terrestres como marinos. Es de este nivel, cuyo espesor máximo alcanza los $150 \mathrm{~cm}$ y el mínimo observable de $35 \mathrm{~cm}$, fue donde se colectó la ictiofauna objeto del presente trabajo.

La ichnofacies Trypanites, en este caso se deduce de manera indirecta a partir de los intraclastos de arcilla azul que por lo común están perforados por bivalvos de la familia Pholadidae, de los cuales es posible aún hallar restos de las valvas dentro de las trazas fósiles de Gastrochaenolites sp. Ello sugiere la existencia de fondos litificados en ambientes marinos en profundidades no mayores a $35 \mathrm{~m}$, asociados a costas rocosas, fondos duros, discordancias y otras superficies de omisión; lo anterior, también se confirma con la presencia de ostras y cirripédios parautóctonos. Sin embargo, es claro que estos fondos duros subacuáticos, poco profundos, se encontraban en áreas aledañas o directamente por debajo de donde se depositó el o los lóbulos sedimentarios del abanico deltaico subacuático, asociado como es lógico a un sistema fluvial.

La energía de la avenida estuvo asociada a uno o varios periodos de lluvias que alcanzaron la tracción necesaria para erosionar los fondos duros y producir los "rip up clasts" o intraclastos, llegando 


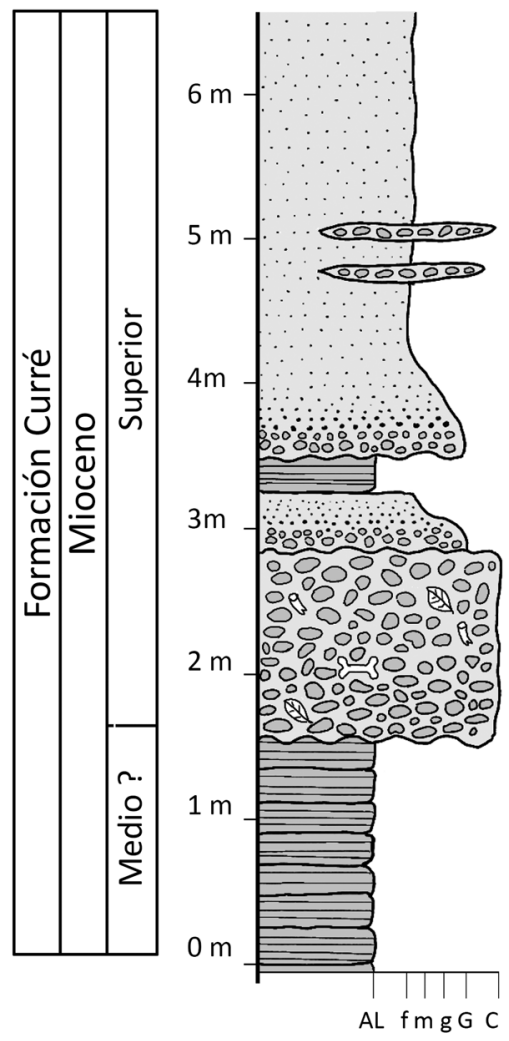

\section{Facies de Bahía Interna o Infralitoral}

Areniscas finas a medias en estratos métricos homogéneos, con difusa laminación paralela y pequeños canales de marea.

Lentes de amplitud centimétrica y longitud métrica con intraclastos retrabajados de arcilla azul.

Estrato retrabajado de arcillas azules.

\section{Facies de Abanico Deltaico Subacuático}

Conglomerado de intraclastos de arcilla azul flotando en una matriz de arcilla y limo, con abundante materia vegetal, restos óseos y dientes de vertebrados continentales y marinos.

\section{Facies de Plataforma Externa o Distal}

Estratos centimétricos de arcillas con una fracción de limo, color azul ricas en glauconita, con laminación milimétrica y abundante detritus vegetal carbonizado y pirita.

Fig. 2: columna estratigráfica y sedimentológica de la Localidad de Limoncito; AL: arcilla - limo; arenisca: "f m g" fina, media y gruesa; $\mathrm{G}$ : grava; C: conglomerado fino.

incluso a desprender fragmentos de estratos de las arcillas azules e integrarlos dentro de los sedimentos gruesos del "fan delta" o abanico deltaico.

\section{C) Bahía Interna próxima a la costa o infrali- toral (Shoreface)}

La litofacies de areniscas finas a medias, bien seleccionadas y limpias, es la litología predominante y conforma un banco de $2.5 \mathrm{~m}$ de espesor, constituido por estratos métricos y homogéneos en el que se observa una difusa laminación paralela y en algunos niveles pequeños "fore sets" de bajo ángulo asociados a pequeños canales de marea. Otros niveles presentan escaso material carbonoso e infrecuentes ichnofósiles. Esta secuencia sobreyace de manera discordante las facies de conglomerado y presenta en su base dos ciclos centimétricos de gravas gruesas que rápidamente gradan a arenas medias y finas. También, se verificó en el campo que esta secuencia se interdigita lateralmente con las facies de abanico deltaico, pues se observó que estas arenas finas a medias llegan a sobreyacer directamente las facies de arcilla azul en algunos puntos.

La ichnofacies Skolitos, en este caso se deduce a partir de pequeñas y escasas trazas de Thalassinoides $s p$. que sugieren un ambiente de alta energía, asociada a sedimentos limpios y bien sorteados con laminación paralela y "fore sets" de bajo ángulo, ello está de acuerdo a la definición dada por Seilacher (1967) y Ekdale et al., 1984) para esta ichnofacies.

\section{PALEONTOLOGÍA}

A continuación se procede a describir las especies de tiburones, rayas y otros ictiolitos. 
Clase CHONDRICTHYES Huxley, 1880

Subclase ELASMOBRANCHII, Bonaparte, 1838

Cohorte EUSELACHII Hay, 1902

Superorden GALEOMORPHII Compagno, 1973

Orden LAMNIFORMES Berg, 1958

Familia LAMNIDAE Müller \& Henle, 1838

Género Isurus Rafinesque, 1810

Isurus desori (Agassiz, 1843) (Sismonda, 1849) Lámina 1(18-19)

Material: dos dientes bien conservados, guardados bajos los códigos CFM-3154 y CFM-3165.

Sinonimia: para un detallado análisis de la sinonimia de la especie I. desori, ver Cappetta (1970), Kruckow \& Thies (1990) y Müller (1992).

Descripción: dientes de mediano tamaño, de contorno triangular y coronas con filos suaves; el CFM-3165 corresponde a un diente inferior de posición lateral intermedia con la corona ligeramente volcada hacia la comisura y hacia atrás; la cara labial es plano convexa y la cara lingual es notablemente convexa con un amplio cuello lingual. La raíz es corta con el lóbulo mesial recto y el lóbulo comisural redondeado, su cara basal es plana, no se observa protuberancia medio lingual y el foramen nutricio abarca toda la altura de la raíz. El CFM-3154, corresponde a un diente superior de posición lateral intermedia, con la cúspide elevada, esbelta, ligeramente volcada hacia la comisura y hacia atrás; la corona presenta un amplio cuello lingual; su cara lingual es notablemente cóncava, en tanto que su cara labial presenta una laxa concavidad. Su raíz es corta con lóbulos de contorno redondeado con una conspicua protuberancia lingual y un angosto canal nutricio.

Discusión: los dientes asignados a la especie Isurus desori, son característicos del género Isurus, sobre todo por poseer raíces, tanto en los inferiores y superiores, con lóbulos mesiales rectos y los lóbulos comisurales bien redondeados; además de cúspides con filos lisos no aserrados. La especie $I$. desori es poco común en el registro fósil y carece de representantes actuales, quizás la característica más notable es la relativa pequeña talla de sus dientes comparados con otras especies de Isurus y en el caso de los dientes superiores en que tengan lóbulos radiculares formado un ángulo relativamente agudo.

Distribución paleogeográfica y bioestratigrafía: la especie Isurus desori (Agassiz, 1843) (Sismonda, 1849), ha sido registrada en el Mioceno de Cuba, Barbados y Venezuela; y el Mioceno inferior de Bonaire (Sánchez-Roig, 1920; Leriche,1938; Casier,1958); fuera del Caribe se ha registrado en el Mioceno de la planicie costera atlántica de Estados Unidos (Leriche, 1942; Müller, 1992), Europa y Japón (Leriche, 1927; Menesini, 1969; Schultz, 1969; Cappetta, 1970; Itoigawa et al., 1985)

Orden CARCHARHINIFORMES Compagno, 1973 Familia HEMIGALEIDAE Hasse, 1879 Subfamilia HEMIPRISTINAE Compagno, 1984

Género Hemipristis Agassiz, 1843

Hemipristis serra Agassiz, 1843 Lámina 1(20)

Sinonimia: para un detallado análisis de la sinonimia de la especie Hemipristis serra Agassiz, 1843, ver Antunes \& Jonet, 1970; Cappetta, 1970 y Laurito, 1999.

Material: un diente lateral superior con la cúspide de la corona rota, código CFM-1874.

Descripción: diente de gran tamaño, de contorno triangular, con corona muy alta y volcada ligeramente hacia la comisura. El filo comisural es fuertemente aserrado, mientras que el filo mesial presenta un aserramiento más fino.

La raíz es amplia y baja con una prominente protuberancia medio lingual que contiene un conspicuo canal mediano.

Discusión: los dientes del género Hemipristis, tanto en la especie actual como en la especie fósil son a nivel morfológico externo indiferenciables. Lo que para muchos autores hace sospechar que ambas denominaciones son sinónimas. Sin embargo, estructuralmente, los dientes de la especie actual Hemipristis elongatus (Klunzinger, 1871), se diferencian de los de la especie fósil por ser de tipo osteodonto, es 
decir que poseen coronas rellenas por osteodentina sin cavidad pulpar y con un muy delgado lecho de ortodentina (cf. Compagno, 1973).

Distribución paleogeográfica y bioestratigrafía: se caracteriza por ser una especie de distribución cosmopolita durante el Cenozoico Superior, siendo una constante su registro en prácticamente todos los yacimientos portadores de dientes fósiles de tiburones en el mundo.

En el territorio nacional, la especie Hemipristis serra Agassiz, se ha reportado para la Formación Punta Judas del Mioceno Medio, la determinación fue hecha por Kruckow (en Seyfried et al., 1985), en la Formación Punta Carballo (Kruckow \& Thies, 1990); ambas en la costa pacífica y en las Formaciónes Uscari y Río Banano en la región Caribe (Laurito, 1999 y Laurito et al., en este número). Otras localidades del Caribe donde se ha registrado la especie Hemipristis serra Agassiz son: la Formación Gatún del Mioceno de Panamá (Gillette, 1984); Venezuela, en las Formaciones Pozón, Socorro, Punta Gavilán y los estratos de Ojo de Agua (Leriche, 1938) y en la Formación Cantaure del Mioceno temprano (Aguilera \& Rodrigues de Aguilera, 2001); Trinidad, en las Formaciones Tamana y Springvale, y en las localidad de Morne Diablo (Casier, 1958). En la Isla de Barbados, aparecen en la Formaciones Oceanic y Bissex Hill (Casier, 1958 y 1966) y en Cuba, en diversas localidades a lo largo de la isla (ver Sánchez Roig, 1920; Iturralde-Vinnent et al., 1994 y 1996).

Hábitat actual y Paleoecología: la especie actual Hemipristis elongatus (Klunzinger, 1871) es un tiburón costero y nectónico en las aguas someras de las plataformas insulares y continentales del océano Indico y Pacífico Occidental. Se alimenta de peces óseos, tiburones y rayas (Compagno, 1984). Fundamentado en ello la especie fósil Hemipristis serra debe ser considerado un tiburón de hábitos nectónicos y epipelágico, habitante de las plataformas continentales e insulares de los mares cálidos y con una distribución pantropical durante el Cenozoico Superior.
Familia Carcharhinidae Jordan \& Everman, 1896

Género Carcharhinus Blainville, 1816

Carcharhinus priscus (Agassiz, 1843)

Lámina 1(1-8)

Sinonimia: para un detallado análisis de la sinonimia de la especie C. priscus (Agassiz, 1843), ver Laurito (1999).

Material: 35 dientes de los cuales 5 son dientes inferiores; códigos CFM-1875-1876, CFM3123-3125, CFM-3127-3131, CFM-3134-3137, CFM-3139, CFM-3141, CFM-3143-3144, CFM3149-3153, CFM-3155, CFM-3164, CFM-3166, CFM-3171-3172 y CFM-3173-3180.

Descripción: dientes de tamaño intermedio a pequeño. Los dientes superiores muestran coronas elevadas de contorno triangular, volcándose hacia la comisura en los dientes laterales proximales. El filo mesial presenta un aserramiento más desarrollado que el filo comisural. La cara lingual de la corona es inflada y en su base posee un cuello marcado pero relativamente angosto. $\mathrm{La}$ raíz es ligeramente más ancha que la corona, con una moderada protuberancia medio lingual y su cara basal es plano cóncava.

Los dientes superiores, presentan cúspides muy angostas, volcadas ligeramente en sentido lingual con filos no aserrados. Las coronas se proyectan lateralmente sobre los lóbulos radiculares, pero no los recubre totalmente. La cara basal de la raíz es ligeramente cóncava.

Discusión: los dientes de C. priscus (Agassiz, 1843) tipifican un morfotipo primordial del género Carcharhinus, caracterizado además por una amplia distribución pantropical que abarcó desde el Oligoceno Superior al Plioceno (Arratia, 1999 y Laurito, 1999), por ello algunos autores consideran que podrían homologarse con varias de las especies actuales que presentan esta morfología básica indiferenciable (Adnet et al., 2007). 
Distribución paleogeográfica y bioestratigrafía: Mioceno de Portugal, España, Polonia, Austria, Italia y sur de Francia (Probst, 1879; Bauzá-Rullan, 1949; Jonet, 1966; Menesini, 1967; Comaschi Caria, 1973; Schultz, 1977 y 1978; Antunes \& Jonet, 1970 y Cappetta, 1970); en el Mioceno Inferior de North Carolina y Mioceno de Maryland (Case, 1980; Müller, 1992); en América del Sur se ha registrado en el Mioceno de Ecuador (Longbottom, 1979) y Perú (De Muizon \& DeVries, 1985) y en América Central, se ha registrado en Costa Rica, en las formaciones Uscari de edad Mioceno Superior-Plioceno Inferior, Punta Judas del Mioceno Medio (Laurito, 1999 y 2004) y de acuerdo al presente trabajo en la Formación Curré de edad Mioceno Superior.

Paleoecología: probablemente y como cualquier otra especie de Carcharhinus, la especie $C$. priscus (Agassiz, 1843), fuera un tiburón tropical costero pelágico de las aguas cálidas y templado cálidas sobre las plataformas continentales e insulares, comunes tanto en aguas someras muy próximas a la costa y el mar abierto, como en áreas próximas al borde de las plataformas.

Carcharhinus longimanus (Poey, 1861)

Lámina 1, (9-16)

Sinonimia: Pterolamiops longimanus (Poey, 1861) in Müller, 1992; p. 54; lám.(5-8 y 12).

Material: 16 dientes, de los cuales 6 son inferiores; códigos CFM-3138, CFM-3142 y CFM3148, CFM-3156-3162, CFM-3167-3170, CFM3174 y 3175 .

Descripción: dientes tipo Carcharhinus de gran tamaño de aproximadamente $2 \mathrm{~cm}$ o más de alto por $2 \mathrm{~cm}$ de ancho. Los dientes superiores presentan coronas triangulares, anchas y elevadas, la porción apical con filos finamente aserrados, abarca la mitad de la altura total del diente; en el caso de los dientes laterales, las coronas se inclinan ligeramente hacia la comisura. La superficie vestibular de la corona es ligeramente convexa, mientras que la superficie lingual es fuertemente convexa, con un marcado cuello de contorno triangular. La raíz es relativamente alta, de contorno rectangular en vista labial y de contorno triangular en vista lingual, con una elevada protuberancia medio lingual, pero esta no es inflada, ni prominente y porta un angosto foramen nutricio.

Los dientes inferiores, presentan contornos triangulares, con coronas altas y con espigadas cúspides finamente aserradas. La superficie vestibular de la corona es plana y varía a ligeramente cóncava, en tanto que la superficie lingual es fuertemente cóncava. El talón comisural es más corto y más redondeado en comparación con el talón mesial que es más recto y ligeramente más alargado.

La raíz es elevada y su cara basal es cóncava con una inconspicua protuberancia medio lingual.

Discusión: los dientes de Carcharhinus longimanus (Poey, 1861) son particular y fácilmente diferenciables de otras especies del género, básicamente por su notable tamaño y por sus raíces rectangulares y elevadas.

Distribución paleogeográfica y bioestratigrafía: Mioceno y Plioceno de Lee Creek Mine, North Carolina y Virginia en Estados Unidos (Müller, 1992) y en América Central, en la Formación Curré, Mioceno Superior de Costa Rica.

Paleoecología: tiburón con un hábitat actual oceánico epipelágico, ocasionalmente costero, frecuentemente en mar abierto en aguas templado cálidas, habitante de las plataformas insulares y continentales (sensu Compagno, 1984).

Género Isogomphodon Gill, 1862

Isogomphodon acuarius (Probst, 1879)

Lámina 1(21)

Sinonimia: Alopecias acuarias n. sp. in Probst, 1879; p. 140, lám. 2(76 y 77). Aprionodon acuarias (Probst) in Cappetta, 1970; p. 57, lám. 17(1-20) 
Para un detallado análisis de la sinonimia de la especie I. acuarius (Probst, 1879), ver Laurito (1999).

Material: un diente lateral anterior de posición incierta; código CFM- 3207.

Descripción: diente de cúspide alta, erguida, asimétrica ligeramente volcada hacia atrás. La cara labial de la corona es suavemente convexa y se proyecta hacia la raíz, recubriendo esta de manera breve y proyectándose en ambas ramas radiculares. Presenta una raíz incompleta y característicamente baja, en comparación con la altura de la corona; además de ser simétrica y bilobulada, con una conspicua protuberancia medio lingual y un amplio foramen nutricio. La cara basal de la raíz es plana y suavemente convexa.

Distribución paleogeográfica y bioestratigrafía: la especie I. acuarius (Probst, 1879) se ha registrado en el Mioceno Inferior de Alemania; Mioceno Medio y Superior de Portugal y sur de Francia (Probst, 1879; Antunes \& Jonet, 1970; Cappetta, 1970); en el Mioceno Inferior de North Carolina (Case, 1980); en el Caribe se ha registrado en el Mioceno Superior-Plioceno Inferior de Costa Rica, Fm. Uscari (Laurito, 1999) y el presente hallazgo del Mioceno Superior de la Fm. Curré, que corresponde al segundo registro de esta especie para Costa Rica y América Central.

Discusión: la especie I. acuarius (Probst, 1879) se diferencia de las otras especies del género, tanto fósiles como actual, por poseer dientes con coronas muy altas y delgadas, y raíces con la cara basal particularmente plana.

Paleoecología: de acuerdo a Laurito (1999), la especie Isogomphodon acuarius (Probst) debe considerarse un tiburón de hábitat costero pelágico de las aguas tropicales y templado cálidas, adyacentes a las zonas continentales e insulares.

Isogomphodon caunellensis (Cappetta, 1970)

Lámina 1(22a-b)

Sinonimia: Aprionodon caunellensis $\mathrm{n}$. sp. in Cappetta, 1970; p. 60; lám. 16(23-27). Isogomphodon caunellensis (Cappetta, 1970) in Cappetta, 1987; p. 121.

Material: un diente lateral anterior de posición presumiblemente superior, de un individuo juvenil; código CFM-3206.
Descripción: diente de corona alta con la mitad apical rota; sin embargo es evidente que la cúspide se vuelca notablemente hacia atrás. $\mathrm{La}$ cara vestibular de la corona es convexa y recubre casi en su totalidad la cara de la raíz y sus lóbulos, en tanto que la cara lingual presenta un característico y amplio collar que limita la corona de la raíz.

La raíz tiene un contorno triangular elevado, con lóbulos amplios y redondos; su área basal es amplia, con un conspicuo foramen nutricio.

Distribución paleogeográfica y bioestratigrafía: Aquitaniano superior, Mioceno Inferior de Caunelle, Montpellier, sur de Francia (Cappetta, 1970 y 1987) y el Mioceno Superior del Sur de Costa Rica.

Discusión: la especie Isogomphodon caunellensis (Cappetta, 1970), básicamente se caracteriza por poseer un cuello lingual muy alto y ensanchado hacia la margen radicular.

Paleoecología: Isogomphodon caunellensis (Cappetta, 1970) es un organismo poco estudiado y su rareza en el registro fósil dificulta deducir su paleoecología.

Familia Sphyrnidae Gill, 1872

Género Sphyrna Rafinesque, 1810

Sphyrna arambourgi Cappetta, 1970

Lámina 1(17)

Sinonimia: Scoliodon taxandriae Leriche in Jonet, 1966; lám. 3(14-15). Cestracion priscus (Ag.) in Menesini, 1967; lám. 6(11). Sphyrna arambourgi n. sp. in Cappetta, 1970; p. 70, lám. 19(1-18). Sphyrna arambourgi Cappetta, 1970 in Cappetta, 1987; p. 127.

Material: un diente superior latero anterior, código CFM-3145.

Descripción: diente triangular de tamaño relativamente grande con aproximadamente $15 \mathrm{~mm}$ de alto, por $15 \mathrm{~mm}$ de ancho.

La corona es relativamente corta, inclinada hacia la comisura, suavemente sigmoidea y se proyecta sobre ambos lóbulos radiculares, pero no llega a cubrir la cara labial de la raíz. Su superficie vestibular es suave y ligeramente convexa; el filo comisural es liso, recto y perpendicular con 
respecto a la línea basal de la raíz; en tanto el filo mesial es liso y ligeramente convexa, casi recta. La raíz es muy corta con el lóbulo comisural roto y el lóbulo mesial casi completo. Su cara basal es plana con un amplio foramen nutricio y carente de protuberancia mediana.

Distribución paleogeográfica y bioestratigrafía: Helvetiano inferior de Montpeyroux, sur de Francia (Cappetta, 1970 y 1987), el Mioceno de Otranto, Puglia, Italia (Menesini, 1967); Portugal (Jonet, 1966) y Mioceno Superior de la Fm. Curré, sur de Costa Rica.

Discusión: los dientes de Sphyrna arambourgi Cappetta, 1970 se diferencian de otras especies del género, básicamente por poseer coronas marcadamente triangulares con filos mesiales laxamente sigmoides y filos comisurales rectos y verticales.

Paleoecología: teniendo en cuenta la ecología actual del género y de acuerdo con Compagno (1984) se puede extrapolar que Sphyrna arambourgi Cappetta, 1970 habitaba las aguas tropicales a templado cálidas, de las plataformas insulares y continentales y de sus aguas adyacentes, no siendo típicos del ambiente oceánico.

Superorden BATOMORPHII Cappetta, 1980 Orden MYLIOBATIFORMES Compagno, 1973 Superfamilia DASYATOIDEA Whitley, 1940

Familia DASYATIDAE Jordan, 1888

Género: Dasyatis Rafinesque, 1810

Dasyatis cavernosa (Probst, 1877) Lámina 2(7)

Sinonimia: Raja cavernosa n. sp. in Probst, 1877, p. 75; lám.1(1-4). Raja rugosa n. sp. in Probst, 1877, p. 76; lám. 1( 6, no las 5 y 7-9). Trygon cavernosus (Probst) in Leriche, 1910, p. 471, fig. 1. Dasyatis cavernosa (Probst) in Cappetta, 1970, p. 88-90, lám. 22(1-13). Dasyatis aff. cavernosa (Probst) in Schultz, 1977, p. 203, lám. 1(1). Dasyatis aff. cavernosa (Probst) in Schultz, 1979, p. 289, tabla 1. Dasyatis cavernosa (Probst) in Case, 1980, p. 94, lám. 9(1a-1e y 2). Dasyatis cavernosa (Probst) in Antunes et al. 1981, p. 19; lám. 4(9). Dasyatis cavernosa (Probst) in Fress, 1991, p. 157; lám. 6(1a-c, 2); lám. 17(6a-b).

Material: un diminuto diente con marcada heterodoncia ginándrica, código CFM-3209.

Descripción: diente con corona de contorno cuadrangular elíptico, no cuspidada y más alta que la raíz. La visera posterior, presenta una superficie lisa que se prolonga en una úvula simple. La superficie oclusal es ornamentada y presenta algunos canales e incisiones que forman la oquedad que caracteriza a la especie. La raíz es bilobulada y los lóbulos son muy amplios.

Discusión: el diente acá descrito es característico del morfotipo de la especie Dasyatis cavernosa (Probst, 1877) y corresponde a una hembra, ya que su corona carece de la cúspide propia de los machos. Por otra parte, este diente es muy pequeño, lo que sugiere que se trata de un elemento juvenil.

Distribución paleogeográfica y bioestratigrafía: Costa Rica es el único país de América Central y el Caribe donde se han descrito varias especies de Dasyatis. Aparte de la Formación Curré y la localidad de Limoncito, la especie D. cavernosa ha sido descrita para el Mioceno Superior - Plioceno Inferior de la Formación Uscari y la localidad de Alto Guayacán (Laurito, 1999), el género se ha registrado en el Medio de la Formación Punta Judas (Laurito, 2004). Fuera del Caribe, Case (1980) registra dicha especie en el Mioceno Inferior de North Carolina. Fuera del Continente Americano, Dasyatis caverno$s a$ (Probst, 1877) es bien conocida en el Oligoceno Superior y el Mioceno de Europa (ver Probst, 1877; Leriche, 1910; Cappetta, 1970; Schultz, 1977 y 1978; Antunes et al. 1981; Fress, 1991).

Paleoecología: se asume el hábitat de esta especie, como el de un organismo béntico sobre los fondos blandos de las plataformas continentales e insulares.

Superfamilia MYLIOBATOIDEA Compagno, 1973 Familia: RHINOPTERIDAE Jordan \& Everman 1896

Género: Rhinoptera Cuvier, 1829

Rhinoptera sp.

Lámina 2(5a-b y 6a-b) 
Material: 2 dientes fragmentados, CFM3187 y CFM-3193

Descripción: dientes de coronas muy gruesas y elevadas de contorno hexagonal, rectas o ligeramente convexas labialmente, pero con superficies oclusales predominantemente planas con bordes rectos y angulares; presentan un cíngulo lingual muy grueso.

Distribución paleogeográfica y bioestratigrafía: el género Rhinoptera ha sido registrado para Costa Rica en la Formación Uscari de edad Mioceno Superior-Plioceno Inferior (Laurito, 1999) y en la Formación Punta Judas, de edad Mioceno Medio (Laurito, 2004). Fuera de Costa Rica, el género Rhinoptera se ha registrado en el Mioceno medio de Panamá en la Formación Gatún (Gillette, 1984). El presente hallazgo, corresponde al cuarto registro de la especie en América Central.

Familia MYLIOBATIDAE Bonaparte, 1838 Género: Myliobatis Cuvier, 1817.

Myliobatis sp.

Lámina 2(1a-b a 3a-b y 4)

Material: 12 dientes, la mayoría corresponden a dientes fragmentados, guardados bajo los códigos CFM-3183-3186, CFM-3188-3189, CFM-3191 y CFM-3194-3199.

Descripción: dientes de contorno hexagonal a oval, coronas oblongas, medianamente elevadas y asimétricas con una ligera pero visible prolongación de los vértices distal y mesial, son convexa lingualmente y de aspecto inflado, siendo más elevada la cara lingual. La cara labial más angosta se proyecta notablemente con una suave concavidad inferior. El límite de la corona con la raíz en la cara lingual está dado por un cíngulo basal muy conspicuo. Las lamelas radiculares son relativamente gruesas, homogéneamente separadas y no se disponen en pares.

Distribución paleogeográfica y bioestratigrafía: en América Central, se han registrado dientes de Myliobatis en la Formación Gatún en el Mioceno Medio de Panamá (Gillette, 1984).
También se han registrado en otras localidades neógenas del Caribe (ver Gillette, 1984; Kruckow \& Thies, 1990; Iturralde-Vinent et al., 1998).

En Costa Rica se han registrado dos morfotipos a nivel específico por Laurito (2004) en la Formación Punta Judas de edad Mioceno Medio.

Género: Aetobatus Blainville, 1816

Aetobatus arcuatus Agassiz (1843) Lámina 2 (8 y 9)

Sinonimia: Aëtobatis arcuatus in Eastman, 1904, p. 76; lám. 29(5). Aetobatis arcuatus in Leriche, 1926, p. 387. Aetobatis arcuatus in Leriche, 1938, p. 8. Aetobatis arcuatus in Zbyszewski \& Moitinho D’Almeida, 1950, p. 56; lám. 8(173) y lám. 9(191). Aetobatus arcuatus in Jonet et al., 1975, pág. 207; lám. 2(21). Aetobatis arcuatus in Brzobohaty \& Schultz, 1978a, p. 422; lám. 1(8). Aetobatus arcuatus in Case, 1980, p. 95, fig. 6B. Aetobatus arcuatus in Gillette, 1984, p. 180. Aetobatus arcuatus in Nolf, 1988, lám. 54(6). Aetobatus arcuatus in Kruckow \& Thies, 1990, p. 68. Aetobatus arcuatus in Müller, 1992, p. 66. Aetobatus arcuatus in Hidden, 1994/1995, p. 73; fig. texto 9; lám. 7(3). Aetobatus arcuatus in Iturralde-Vinent et al., 1998, p. 142; fig. 5:1-9. Aetobatus sp. in Purdy et al., 2001, p. 94.

Material: cuatro dientes inferiores, fragmentados depositados en el Museo Nacional de Costa Rica, bajo los códigos CFM-3181, CFM-3182, CFM-3190 y CFM-3192.

Descripción: dientes hexagonales alongados con coronas bajas, delgadas y carentes de ornamentación. La raíz en estos dientes, son mucho más altas y anchas que sus coronas y presentan lamelas dispuestas de manera inclinada con respecto al borde labial o lingual de las coronas.

Distribución paleogeográfica y bioestratigrafía: en Costa Rica, Kruckow (1975) y Kruckow \& Thies (1990) registran la especie $A$. arcuatus Agassiz (1843) en la Formación Punta Carballo de edad Mioceno Medio en la costa pacífica y en la localidad de Bonilla a orillas del río Reventazón de probable edad Mioceno Superior- 
Plioceno Inferior en la vertiente del Caribe. Laurito (2004), ha registrado esta especie en la Formación Punta Judas del Mioceno Medio de la región Pacífico central.

En el resto de América Central, Gillette (1984) registra A. arcuatus Agassiz (1843) en la Formación Gatún del Mioceno Medio de Panamá. En la cuenca del Caribe, Leriche (1938), registra dicha especie en la Formación La Vela del Mioceno Superior de Venezuela e Iturralde-Vinent et al. (1998) en el Mioceno inferior Tardío de Cuba. En América de Norte, son comunes los registros en el Mioceno de Maryland, Virgina y Carolina del Norte (Eastman, 1904; Leriche, 1942; Case, 1980; Kruckow, 1983; Kruckow \& Thies, 1990; Müller, 1992). En América del Sur se ha registro material fragmentario de Aetobatus que podría ser atribuido eventualmente a esta especie, en el Mioceno de Ecuador (Longbottom, 1979), Mioceno de Perú (Apolín et al., 2004) y Mioceno Superior - Plioceno Inferior de Chile (Long, 1993).

\section{DISCUSIÓN}

\section{Diferencias dentales entre los géneros Myli- obatis, Rhinoptera y Aetobatus}

Las diferencias entre los géneros arriba mencionados, han sido establecidas con base en las siguientes características dentales.

A. El género Rhinoptera se diferencia de Myliobatis por poseer coronas muy altas, con superficies oclusales de bordes rectos y angulares y la presencia de un cíngulo lingual muy grueso.

B. El género Myliobatis se caracteriza por coronas medianamente elevadas, infladas y de contornos con vértices redondeados.

C. El género Aetobatus, se diferencia de los géneros anteriores por poseer raíces con lamelas dispuestas de forma oblicua y no perpendicular al borde anterior o posterior del diente. También se diferencia por poseer corona muy bajas, de menor altura que Myliobatis, en particular si se compara con la altura de la raíz. Se asume que para el Mioceno y el Plioceno el género es monoespecífico, como en el caso de la especie actual o corresponde con un morfotipo nominado Aetobatus arcuatus Agassiz (1843).

Paleoecología de los géneros Myliobatis, Rhinoptera y Aetobatus: en términos generales y teniendo en cuenta la ecología actual de dichos géneros, deben ser considerados animales bentónicos, habitantes de las plataformas continentales, pelágico costeros y pelágico oceánicos de los mares tropicales a templado cálidos. Su alimentación consiste de moluscos (bivalvos y gasterópodos) y crustáceos.

\section{OTROS ICTIOLITOS}

Clase OSTEICHTHYES

Subclase ACTINOPTERYGII

Division TELEOSTEI

Superorden ACANTHOPTERYGII

Orden PERCIFORMES

Suborden PERCOIDEI

\section{Familia SPARIDAE}

Género: Sparus

Sparus sp.

Lámina 2(10 y 11)

Material: 2 dientes cónicos depositados bajo los códigos CFM-3208 y CFM-3210.

Descripción: dientes de contorno basal circular, con corona cónica, inflada, elevada y carente de un cíngulo basal, su cara basal es convexa.

Distribución paleogeográfica y bioestratigráfica: es la tercera vez que se registra como fósil el género Sparus en Costa Rica, fuera del presente hallazgo, Laurito (1999), describe Sparus especie indeterminada en la localidad de Alto Guayacán, Mioceno Superior-Plioceno Inferior de la Fm. Uscari y en el Mioceno Superior de la Fm. Río Banano (Laurito et al., en este número). En el Caribe, Casier (1958) describe dientes del género Sparus del Eoceno de la Isla de Barbados. Por otra parte Gillette (1984) hace referencia al hallazgo de Sparidae en la Formación Gatún en el Mioceno Medio de Panamá. Fuera del Caribe, son numerosos los hallazgos del género en el Mioceno de Europa. 
Discusión: los dientes de la familia Sparidae muestran una conspicua heterodoncia, variando desde formas caniniformes o molarifornes, dependiendo de la estrategia alimentaria, ello tiene aplicación a nivel sistemático genérico de acuerdo con Otero \& Gayet (2001). En el caso de las formas halladas en la Fm. Curré, solo se puede decir que se trata del género Sparus.

Paleoecología: de acuerdo a Cervigón \& Fischer (1979), las especies de Sparidae habitan en la actualidad las plataformas continentales y son organismos bentónicos de fondos duros.

\section{Espinas caudales de rayas \\ Lámina 2 (12 y 13)}

Material: 6 fragmentos de espinas de rayas, depositadas bajo los códigos CFM-3200 a CFM3205 en el Museo Nacional de Costa Rica.

Descripción: fragmentos de espinas aserradas comprimidas dorsoventralmente, con el extremo de los aserramientos dirigidos hacia la base de la espina. En corte transversal son planas basalmente y convexas dorsalmente. La cara dorsal puede presentar dos canales longitudinales o carecer de ellos.

Discusión: es difícil establecer con seguridad a que especie o al menos a que género pertenecen estos ictiolitos, ya que podrían ser de un dasiátido, un rinoptérido o de un miliobátido.

\section{INTERPRETACIÓN PALEOECOLÓGICA}

Las especies descritas son en general habitantes de las plataformas insulares y continentales en mares tropicales con salinidad normal. Pero, si se analiza en detalle el hábitat de cada género y su ontogenia, es posible deducir ciertos hábitos y condiciones del ambiente marino que permiten explicar el ensamble de especies recuperado en la litofacies de conglomerado fino, interpretado como un abanico deltaico subacuático, donde fueron halladas.

Las especies de Carcharhinidae descritas, en general, sugieren formas que podían ingresar a aguas poco profundas en las áreas costeras, incluso aquellas especies predominantemente pelágicas como la especie Carcharhinus longimanus
(Poey, 1861) que eventualmente se aventura a aguas someras de las plataformas continentales e insulares.

También es probable que algunas de estas formas fósiles de carcahrhínidos acá descritas toleraran las bajas salinidades e incluso ingresaran a ríos y estuarios. Ello lo confirman las formas juveniles de dos especies de Isogomphodon, cuyos representantes actuales del género, son costeros y suelen ingresar a ríos y estuarios para su alimentación y reproducción. Esto también sería acorde con el hallazgo de un ejemplar juvenil de Dasyatis cavernosa (Probst, 1877).

Por su parte, el registro del Hemigaleidae Hemipristis serra, representado por un diente de un individuo adulto de gran talla; no contradice el ambiente somero deducido a partir de la litología y la sedimentología de los conglomerados donde se recuperaron los fósiles, pues los representantes actuales del género, ingresan a aguas tan someras con apenas un metro de profundidad (Compagno, 1984).

En cuanto a la presencia de dientes de Rhinopteridae y Myliobatidae, son organismos bentónicos, cuya dieta consiste de moluscos y son visitantes habituales de las aguas someras de las plataformas y los fondos blandos y duros del nerítico, ricos en ostras y bivalvos tanto sésiles como epi y endo bentónicos, por lo que su asociación con los sedimentos conglomerádicos del abanico deltaico pudo deberse al acarreo originado por la sedimentación de alguno de los lóbulos.

\section{EDAD DE LA FAUNA}

Los peces cartilaginosos descritos, son típicos del Mioceno, sin embargo la fauna de animales terrestres, en especial la presencia del mastodonte Rhynchohterium blicki, sugieren una edad tentativa Mioceno Superior (ver Laurito \& Valerio, 2005).

\section{CONCLUSIONES}

Se describe la ictiofauna colectada en la localidad fosilífera de San Gerardo de Limoncito, la cual se ubica en el tope de la Formación Curré de 
edad Mioceno Medio a Superior. La fauna se recuperó de litofacies de conglomerado fino depositado en un ambiente marino próximo a la costa, asociado a facies de abanicos deltaicos subacuáticos o "fan delta's sediments".

La ictiofauna está compuesta principalmente por dos superordenes de Chondrichthyes, los Galeomorphii, representados por 2 ordenes: 1. Lamniformes con 1 familia, Lamnidae y una especie I. desori (Agassiz, 1843) (Sismonda, 1849); 2. Carcharhiniformes con 3 familias: Hemigaleidae con una especie Hemipristis serra Agassiz, 1843; Carcharhinidae con 4 especies, Carcharhinus priscus (Agassiz, 1843), Carcharhinus longimanus (Poey, 1861), Isogomphodon acuarius (Probst, 1879) e Isogomphodon caneullensis (Cappetta, 1970) y Sphyrnidae con una especie Sphyrna arambourgi Cappetta, 1970, para un total de 7 especies; y los Batomorphii con un orden Myliobatiformes, representados por 3 familias, Dasyatidae con una especie, Dasyatis cavernosa (Probst, 1877); Rhinopteridae con una especie indeterminada y los Myliobatidae con la especie Aetobatus arcuatus Agassiz (1843) y una especie indeterminada de Myliobatis; para un total de 4 géneros y 4 especies. Además se describen algunas espinas de raya cuya asignación genérica no es posible develar. Para un total de 11 especies de Chondrichthyes y 1 de Osteichthyes.

Además, se registra y describe una familia de Osteichthyes, Sparidae, representada por el género Sparus, especie indeterminada.

La fauna de peces cartilaginosos de San Gerardo de Limoncito es característica del Mioceno, ya que presenta elementos típicos del Thetis, es decir especies cosmopolitas y pantropicales. Pero además, comparte todas las especies descritas a excepción de Carcharhinus longimanus (Poey, 1861), con la fauna paleo mediterránea de la Región de Montpellier en el sur de Francia, descrita por Cappetta (1970). Lo más sobresaliente es que por primera vez se describe una especie que se consideró por mucho tiempo endémica de Montpellier, la especie Isogomphodon caneullensis (Cappetta, 1970).

La recuperación de restos de ostras, cirripédios e ichnofósiles de Gastrochaenolites sp., apoya la tesis de la existencia de "hard grounds" o fondos endurecidos sublitorales y estos fondos corresponden al hiato existente entre las arcillas azules y las facies de abanico deltaico, las trazas de Pholadidae sugieren profundidades no mayores a los $35 \mathrm{~m}$.

La extensión de la facies de abanico deltaico abarca un área de al menos 5 kilómetros de radio, aunque es probable que se extienda mucho más, ello implica que el abanico deltaico, está constituido por varios lóbulos sedimentarios y que el antiguo río que lo alimentaba drenaba una cuenca importante, extendiéndose sus sedimentos ampliamente dentro de la zona sublitoral.

Por último, la asociación de peces analizada define el ecosistema marino del yacimiento de San Gerardo de Limoncito.

\section{REFERENCIAS}

ADNET, S., ANTOINE, P.O., HASSAN BAQRI, S.R., CROCHET, J.Y., MARIVAUX, L., WELCOMME, J.L. \& MÉTAIS, G., 2007: New tropical carcharhinids (chondrichthyes, Carcharhiniformes) from the late Eocene-early Oligocene of Balochistan, Pakistan: Paleoenvironmental and paleogeographic implications.- Journal of Asian Earth Sciences, 30: 303-323.

AGUILERA, O. \& RODRIGUES DE AGUILERA, D., 2001: An Exceptional Coastal Upwelling Fish Assemblage in the Caribbean Neogene.- America Journal of Paleontology, 75(3): 732-742.

ANTUNES, M.T. \& JONET, S., 1970: Requins de l'Helvétien Supérieur et du Tortonien de Lisbonne.- Rev. Fac. Ciênc. Lisboa, 2da. Sér. C; XVI(1):119-280.

ANTUNES, M.T., JONET, S. \& NASCIMENTO, A., 1981: Vertébrés (crocodiliens, poissons) du Miocène marine de l'Algarve occidentale.- Ciênc. da Terra (UNL), Lisboa, 6:9-38.

APOLÍN， J., GONZÁLEZ-BARBA， G. \& MARTÍNEZ, J., 2004: Seláceos del Mioceno Superior de Quebrada Pajaritos, Piura, Perú.- XII Congreso Peruano de 
Geología. Resúmenes extendidos. Sociedad Geológica del Perú: 401-404.

ARRATIA, G., 1999: Contributions of the Southern South America to vertebrate paleontology.- Münchner Geowissenschaftliche Abhandlungen, 30A: 1-342.

BRZOBOHATY, R. \& SCHULTZ, O., 1978: Die Fischfauna des Badenien. - En: Brestenská, E. (ed.): Chronostratigraphie und Neostratotypen, Miozän der Zentralen Paratethys.- Slowakischen Akademie der Wissenschaften, 441-465.

BAUZA-RULLÁN, J., 1949: Contribuciones al conocimiento de la fauna ictiológica fósil de Mallorca.- Bol. Soc. Española Hist. Nat. 47:203-217.

CASE, G.R., 1980: A selachian fauna from the Trent Formation, Lower Miocene (Aquitanian) of Eastern North Carolina.- Palaeontographica Abt. A. 171(1-3):75-103.

CASIER, E., 1958: Contribution à l'étude des poissons fossiles des Antilles.- Mém. Suisses de Paléontologie, 74:1-95.

CASIER, E., 1966: Sur la fauna ichthyologique de la Formation Bissex Hill et de série océanique de l'Ile de la Barbade, et sur l'âge de ces formations.- Eclogae geol. Helvetiae, 59(1):493-516.

CAPPETTA, H., 1970: Les sélaciens du Miocène de la région de Montpellier.Paleovertebrata, Mém. Extr. 1970, 1-139.

CAPPETTA, H., 1987: Chondrichtyes II: Mesozoic and Cenozoic Elasmobranchii.En: SCHULTZE, H.P. (ed.): De Handbook of Paleoichthyology, Vol. 3B: 1-193.

CERVIGÓN, F. \& FISCHER, W., 1979: Catálogo de especies marinas de interés económico actual o potencial para América Latina. Parte I: Atlántico centro y suroccidental.372 págs. Infopesca.
CHAFETZ, H.S. \& REID, A., 2000: Syndepositional shallow-water precipitation of glauconite minerals.- Sedimentarie Geology, 136:29-42.

COMASCHI CARIA, I., 1973: I pesci del Miocene delle Sardegna. Cagliari, Stab. Tipo.- 33 págs. Ed. Fossataro.

COMPAGNO, L.J.V., 1973: Interrelationships of living elasmobranchs. - En: GREENWOOD, P.H., MILES, R.S. \& PATERSON, C. (eds.): Interrrelationships of fishes. - Journal Linn. Soc. (Zool.) 53 Suppl. 1:15-61.

COMPAGNO, L.J.V., 1984: FAO species catalogue. Vol. 4, Sharks of the world. An annotaded and illustrated catalogue of sharks species known to date. Part 1- Hexanchiformes to Lamniformes \& Part 2- Carcharhiniformes.- FAO Fish. Synop. (125) Vol.4, Pt.1: 1-249 - Pt.2: 251-655.

DE MUIZON, C. \& DE VRIES, T.J., 1985: Geology and paleontology of Late Cenozoic marine deposits in the Sacaco area.- Geologische Rundschau, 74(3):547-563.

DENGO, G., 1962: Estudio Geológico de la Región de Guanacaste, Costa Rica.- 112 págs. IGN, San José.

EASTMAN, C.R., 1904: Pisces. In Miocene (of Maryland) Systematic Paleontology.Maryland Geol. Survey: 71-93.

EKDALE, A.A, BROMLEY, R.G. \& PEMBERTON, S.G., 1984: Ichnology traces fossils in sedimentary and Stratigraphy.Soc. Econ. Paleontol. Minerals., Short Course Notes, 15: 1-137.

FREESS, W.B., 1991: Elasmobranchii und Teleostei des Stemberger Gestesins (Oberoligozän).- Arch. Geschiebekde, 1(3/4):129-216. 
GILLETTE, D.D., 1984: A marine ichthyofauna from the Miocene of Panama, and the Tertiary Caribbean Faunal Province.- En: MACFADDEN, B.J. (ed.): Origin and evolution of the Cenozoic Vertebrate Fauna of Middle America.- Journal of Vertebrade Paleontology, 4(2):172-186.

HENNINGSEN, D., 1965: La Fila Costeña del Pacífico y su posición dentro del sistema montañoso centroamericano meridional.90 págs., Giessen.

ITOIGAWA, J., NISHIMOTO, H., KARASAWA, H. \& OKUMURA, Y., 1985: Miocene fossils of the Mizunami group, Central Japan. 3. Elasmobranchs.- Monogr. Mizunami Fossil Museum, 5:1-99.

ITURRALDE-VINENT, M., HUBBELL, G. \& ROJAS, R., 1994: Cuban Fossil Sharks.- En: DONOVAN, S.K. (ed.): Geological and Biological Evolution of the Caribbean Region.- Contributions to Geology, UWI 1:22-23.

ITURRALDE-VINENT, M., HUBBELL, G. \& ROJAS, R., 1996: Catalogue of Cuban fossil Elasmobranchii (Paleocene to Pliocene) and paleogeographic implications of their Lower to Middle Miocene occurrence.Jour. Geol. Soc. Jamaica, 31:7-21.

ITURRALDE-VINENT, M., LAURITO, C., ROJAS, R. \& GUTIÉRREZ, M.R., 1998: Myliobatidae (Elasmobranchii: Batomorphii) del Terciario de Cuba.- Rev. de la Soc. Mexicana de Paleontología, 8(2):135-145.

JONET, S., 1966: Notes d'Ichthyologie miocene. II Note. Les Carcharhinidae.- Bol. Mus. Lab. Miner. Geol. Fac. Cienc. Lisboa, 10 (2):65-88.

KRUCKOW, TH., 1975: Haifisch-Zähne als präkolumbianische Werkseuge aus Costa Rica (Mittelamerika) und Nordamerika .Jb. Wittheit Bremen, 19:191-197.
KRUCKOW, TH., 1983: Ambiatlantische Beziehungen der tertiären Elasmobranch Faunen.- Zbl. Geol. Paläont. 1(3) : 213-222.

KRUCKOW, TH. \& THIES, T., 1990: Die Neoselachier der Paläokaribik (Pisces: Elasmobranchii).- Cour. Forsch. Inst. Senckenberg, 119:1-102.

LAURITO, C., 1999: Los seláceos fósiles de la localidad de Alto Guayacán (y otros ictiolitos asociados), Mioceno Superior Plioceno Inferior de la Formación Uscari, provincia de Limón, Costa Rica.-168 págs. LAURITO, C (ed.), San José.

LAURITO, C., 2004: Ictiofauna de la Formación Punta Judas, Mioceno Medio, provincia de Puntarenas, Costa Rica.- Brenesia, 62: 57-74.

LAURITO, C. \& VALERIO, A.L., 2005: First record of Rhynchotherium blicki (Frick, 1933) for the Late Cenozoic of Costa Rica.- Revista Geológica de América Central, 33:75-82.

LAURITO, C., VALERIO, A.L., OVARES, E., HERNÁNDEZ, A.C. \& PIZARRO, D., en este número: Peces fósiles de la localidad Lomas de Siquirres, cauce del río Reventazón, Formación Río Banano, Mioceno Superior, provincia de Limón, Costa Rica.- Revista Geológica de América Central.

LERICHE, M., 1910: Sur quelques poissons du Crétacé du Basin de Paris, Note sur les poissons Néogènes de la Catalogne.Bull. Soc. Géol. France, 4 sér. X: 45:471474.

LERICHE, M., 1926: Les poissons tertiaires de la Belgique IV: Les poissons néogènes.- Mém. Mus. Hist. Nat. Belgique, 32:364-472.

LERICHE, M., 1927: Les poissons de la Mollase Suisse.- Mém. Soc. Paléont. Suisse, 46 (1):1-28. 
LERICHE, M., 1938: Contribution à l'étude des poissons fossiles des pays riverains de la Méditerranée américaine (Vénézuéla, Trinité, Antilles, Mexique).- Mém. Soc. Paléont. Suisse, 41:1-42.

LERICHE, M., 1942: Contribution à l'étude des faunes ichthyologiques marines des terrains tertiaires de la Plaine Cotiere Atlantique et du centre des États-Unis. Le synchronisme des formations tertiaires des deux cotés de L'Atlantique.- Mém. Soc. Géol. France, Paris (n.s.) 45:1-110.

LOHMANN, W., 1934: Stratigraphie und Tektonik des Hochlandes von Costa Rica.Geol. Rundschau, 25: 10-26.

LONG, D.J., 1993: Late Miocene and Early Pliocene fish assemblages from the north central coast of Chile.- Tertiary Research, 14(3):117-126.

LONGBOTTOM, A.E., 1979: Miocene sharks' teeth from Ecuador.- Bull. Br. Mus. nat. Hist. (Geol.) 32(1):57-70.

MENESINI, E., 1967: Ittioliti Pliocenici di Porto Craulo (Otranto).- Atti. Soc. toscana Sci. Nat., Pisa, Ser. A, 74: 1-20.

MENESINI, E., 1969: Ittiodontoliti Miocenici di Terra d'Otranto (Puglia). Paleontograf. Italica, 65 (n.ser., 35): 1-61.

MORA, S., 1979: Estudio geológico de una parte de la región sureste del Valle del General, provincia de Puntarenas, Costa Rica.- 157 págs. Univ. de Costa Rica, San José [Tesis de Lic.].

MÜLLER, A., 1992: Ichthyofaunen aus dem Atlantischen Tertiär der USA. Systematik, Paläoökologie, Biostratigraphie, Evolution undPaläobiogeographie.-Habilitationschrift im Fachbereich Geowissenschaften der Westfälischen Whilhelms. Universität Münster.
NOLF, D., 1988: Dents de Requins et Raies du Tertiaire de la Belgique. Edition de l'Institut Royal des Sciences naturelles de Belgique.- 184 págs. Bélgica.

PROBST, J., 1877: Beiträge zur Kenntniss der fossilen Fische aus der Molasse von Baltringen. II. Batoidei.- Jh. Ver. Vaterländ. Naturkde. Württemberg, 33:69-103.

PROBST, J., 1879: Beiträge zur Kenntniss der fossilen Fische aus der Molasse von Baltringen. Hayfische.- Jh. Ver. Vaterländ. Naturkde. Württemberg, 35:127-191.

OTERO,O.\&GAYET,M.,2001:Palaeoichthyofaunas from the Lower Oligocene and Miocene of the Arabian Plate: palaeoecological and palaeobiogeographical implications.Palaeogeography, Palaeoclimatology, Palaeoecology, 165: 141-169.

PURDY, R.W., SCHENEIDER, V.P., APPLEGATE, S.A., MCLELLAN, J.H., MEYER, R.L. \& SLAUGHTER, B., 2001: The Neogene Sharks, Rays, and Bony Fishes from Lee Creek mine, Aurora, North Carolina.- En: RAY, C.E. \& BOHASKA, D.J. (eds.): Geology and Paleontology of the Lee Creek mine, North Carolina, III.- Smithsonian Contributions to Paleobiology, 90: 71-202.

SANCHEZ-ROIG, M., 1920: Escuálidos del Mioceno de la Habana.- Bol. Minas, 6: 1-16.

SCHULTZ, O., 1969: Die Selachierfauna (Piscis, Elasmobranchii) aus den Phosphoritsanden (Unter-Miozän) von Plesching bei Linz.Oberösterreich. Naturk. Jahr der Stadt Linz. 61-102.

SCHULTZ, O., 1977: Elasmobranch and teleost fish remains from Korytnica Clays (Middle Miocene; Holy Cross Mountains, Poland).- Acta Geologica Polonica, 27(2):201-209.

SCHULTZ, O., 1978: Neue und fehlinterpretiere Fischformen aus dem Miozän des Wiener 
Beckens.- Ann. Naturhistor. Mus. Wien. 81:203-219.

SEILACHER, A., 1967: Bathymetry of trace fossils.- Marine Geology, 5:413-429.

SEYFRIED, H., SPRECHMANN, P. \& AGUILAR, T., 1985: Sedimentología y paleoecología de un estuario del litoral
Pacífico del Istmo Centroamericano primordial (Mioceno Medio, Costa Rica).Revista Geológica de América Central, $3: 1-68$.

ZBYSZEWSKI, G. \& MOITINHO D’ALMEIDA, F., 1950: Os peixes miocénicos portugueses.- Comun. Serv. Geol. Portugal, 31:309-412. 


\section{LÁMINA 1}

Carcharhinus priscus (Agassiz, 1843). 1: diente superior lateral anterior en vista vestibular (CFM1875). 2: diente superior lateral anterior en vista vestibular (CFM-3171). 3: diente superior lateral posterior en vista vestibular (CFM-3166). 4: diente superior lateral posterior en vista vestibular (CFM-3164). 5: diente superior lateral posterior en vista vestibular (CFM-1876). 6: diente inferior próximo a la sínfisis en vista vestibular (CFM-3179). 7: diente inferior lateral posterior en vista vestibular (CFM-3176). 8: diente inferior lateral posterior en vista vestibular (CFM-3180).

Carcharhinus longimanus (Poey, 1861). 9: diente superior lateral intermedio en vista vestibular (CFM-3157). 10: diente superior lateral Intermedio en vista vestibular (CFM-3160). 11: diente superior lateral anterior en vista vestibular (CFM-3156). 12: diente superior lateral posterior en vista vestibular (CFM-3161). 13: diente inferior próximo a la comisura en vista vestibular (CFM-3170). 14: diente inferior lateral anterior en vista vestibular (CFM-3169). 15: diente inferior lateral anterior en vista vestibular (CFM-3167). 16: diente inferior próximo a la comisura en vista vestibular (CFM-3168). 3145).

Sphyrna arambourgi Cappetta, 1970. 17: diente inferior lateral anterior en vista vestibular (CFM-

Isurus desori (Agassiz, 1843) (Sismonda, 1849). 18: diente inferior lateral próximo a la comisura en vista vestibular (CFM-3165). 19: diente superior lateral posterior en vista vestibular (CFM-3154). 1874).

Hemipristis serra Agassiz, 1843. 20: diente superior lateral intermedio en vista lingual (CFM-

Isogomphodon acuarius (Probst, 1879). 21: diente lateral anterior de posición incierta en vista vestibular (CFM-3207).

Isogomphodon caunellensis (Cappetta, 1970). 22: diente lateral posterior, a- vista vestibular y bvista labial (CFM-3206).

Escala visual $=10 \mathrm{~mm}$, excepto para 21 y $22=1.0 \mathrm{~mm}$. 


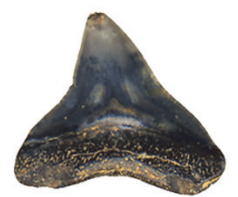

1

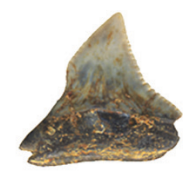

5

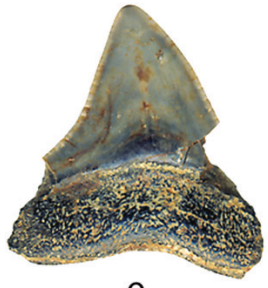

9

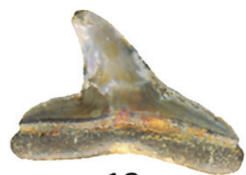

13

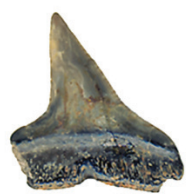

17

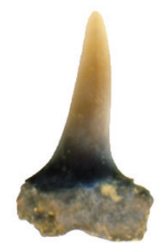

21

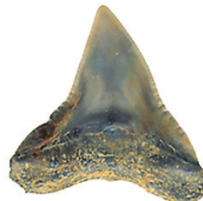

2

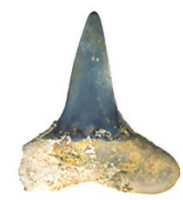

6

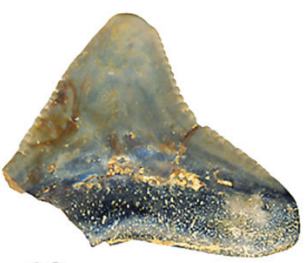

10

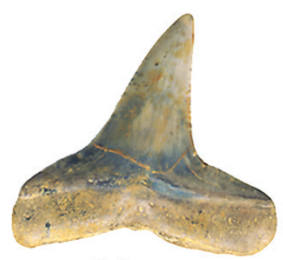

14

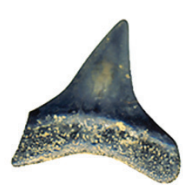

18

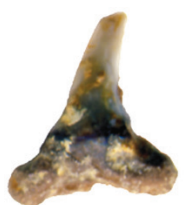

$22 a$

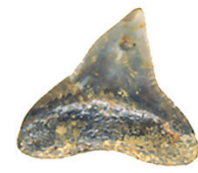

3

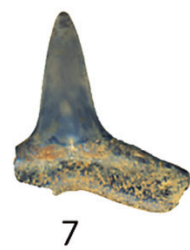

11
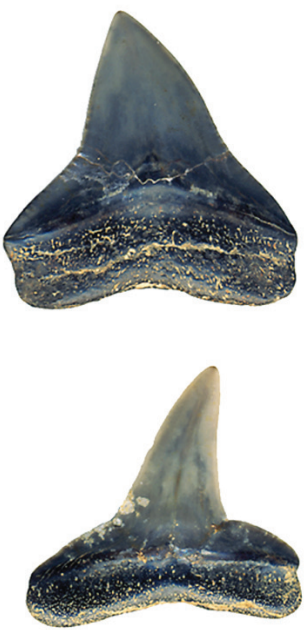

15

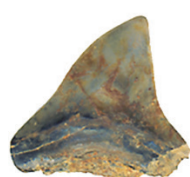

4

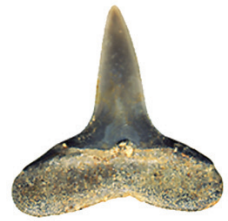

8

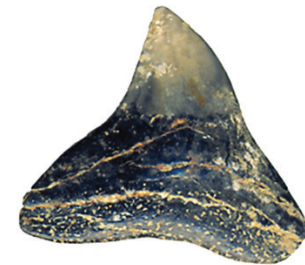

12

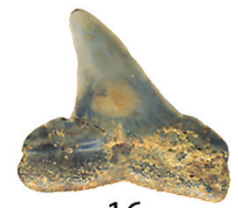

16

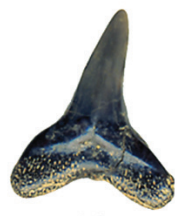

19

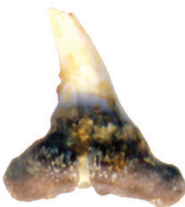

$22 a$

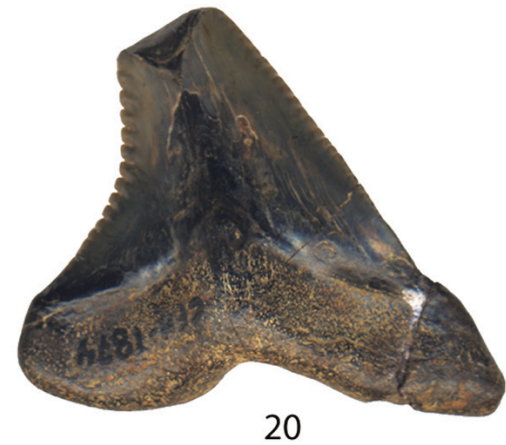

20 


\section{LÁMINA 2}

Myliobatis sp. 1: Diente mediano de posición incierta a-vista anterior y b-vista oclusal (CFM-3189). 2: Diente mediano de posición incierta a-vista anterior y b-vista oclusal (CFM-3191). 3: Diente mediano de posición incierta a-vista anterior y b-vista oclusal (CFM-3196). 4: Diente mediano de posición incierta en vista oclusal (CFM-3199).

Rhinoptera $s p$. 5: Diente mediano de posición incierta a-vista anterior y b-vista oclusal (CFM3187). 6: Diente mediano de posición incierta a-vista posterior y b-vista oclusal (CFM-3193).

Dasyatis cavernosa (Probst, 1877). 7: Diente de posición incierta en vista frontal(CFM-3209).

Aetobatus arcuatus (Agassiz, 1843). 8: Diente mediano de posición incierta en vista basal (CFM3190). 9: Diente mediano de posición incierta en vista basal (CFM-3192).

Sparus sp. 10: Diente de posición incierta (CFM-3208). 11: Diente de posición incierta (CFM3210). 12 y 13: Espinas caudales de Myliobatiformes indeterminados (CFM- 3204 y CFM-3202)

Escala visual $=10 \mathrm{~mm}$, excepto para 7,10 y $11=1.0 \mathrm{~mm}$. 


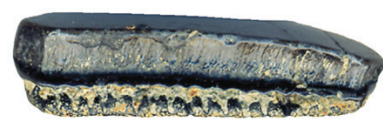

$1 \mathrm{a}$

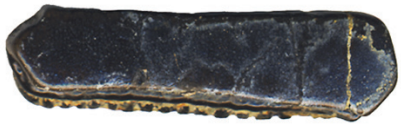

$1 \mathrm{~b}$

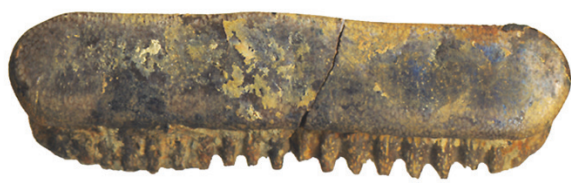

4

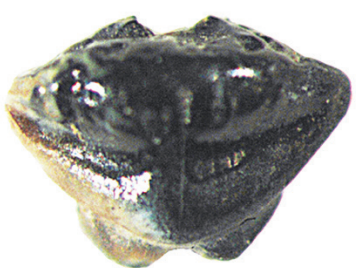

7

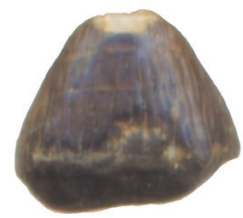

10

11

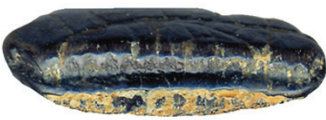

$2 \mathrm{a}$

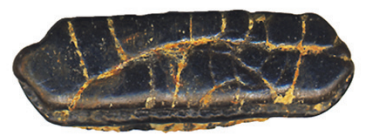

$2 b$

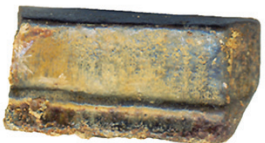

$5 a$

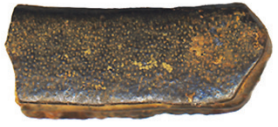

$5 b$

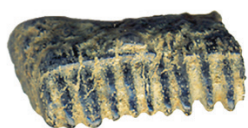

8

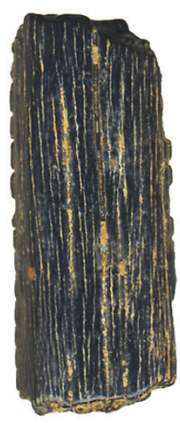

12

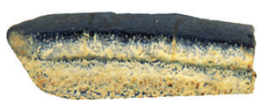

$3 a$

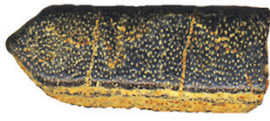

$3 b$

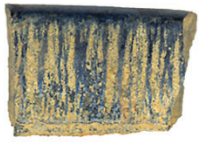

$6 a$

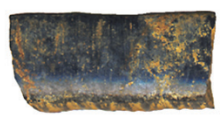

$6 b$

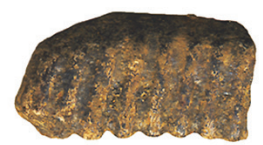

9

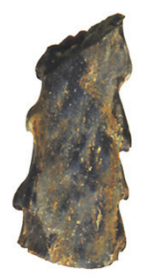

13 
